\title{
Cross-circulation thrombectomy through posterior communicating artery for acute middle cerebral artery occlusion using Solitaire FR stent with intermediate catheter assisting technique
}

\author{
Di Li ${ }^{1}$, Zhongjun Chen ${ }^{1}$, Tieping Fan ${ }^{1}$, Xusheng Zhao ${ }^{1}$, Hengxu $\mathrm{Qi}^{1}$, Xuesong Liang ${ }^{2}$, \\ Aline M. Thomas ${ }^{3}$, Shen $\mathrm{Li}^{2}$ \\ ${ }^{1}$ Department of Neurointervention, Dalian Municipal Central Hospital affiliated with Dalian Medical University, Dalian, China \\ ${ }^{2}$ Department of Neurology, Dalian Municipal Central Hospital affiliated with Dalian Medical University, Dalian, China \\ ${ }^{3}$ Russell H. Morgan Department of Radiology and Radiological Sciences, Johns Hopkins University School of Medicine,
}

Baltimore, USA

Key words: cross-circulation, acute ischaemic stroke, thrombectomy, swim technique

(Neurol Neurochir Pol 2021; 55 (4): 403-406)

\section{To the Editors}

Cross-circulation thrombectomy gains access to emergent large-vessel occlusions without a favourable direct route through the anterior or posterior communicating artery. Although there have been a few successful cases reported, this approach involves long distance navigation of the endovascular devices in the tortuous Circle of Willis collaterals, accompanied by a high risk of clots escaping and arterial injury.

We here present the first case of cross-circulation thrombectomy with a good outcome for acute middle cerebral artery occlusion and chronic ipsilateral internal carotid artery occlusion via the patent posterior communicating artery using the Solitaire FR/Stent With Intermediate Catheter Assisting (SWIM) technique. We demonstrate that cross-circulation thrombectomy provides an opportunity for recanalisation of occluded arteries with unfavourable direct access or anatomical constraints. The SWIM technique, which employs an intermediate catheter, is beneficial in minimising mechanical injuries and reducing exposure of the retrieved clot to the bloodflow, thus lessening complications.

Acute occlusion of the middle cerebral artery (MCA) with tandem occlusion of ipsilateral internal carotid artery (ICA) has a low recanalisation rate after intravenous thrombolysis, and a poor prognosis [1]. There are currently no treatment guidelines for these patients, and only a few case studies have reported successful cross-circulation thrombectomy through the Willis circle, via trans-anterior or posterior communicating artery (AComA or PComA) routes [2-5].

However, the endovascular devices have to travel long distances in the tortuous intracranial arteries using this approach, thereby increasing the risk of mechanical injury, the escape of clots, and thromboembolisms [6].

The newly developed Solitaire FR/Stent With Intermediate Catheter Assisting (SWIM) technique employing an intermediate catheter could potentially reduce trans-circulation thrombectomy-associated complications.

A 63-year-old male with a 40-year history of smoking, alcohol consumption (30 years), hypertension and coronary artery disease was transferred to the emergency department 90 minutes after the acute onset of somnolence, right hemiplegia, dysarthria, right facial droop, visual field defect, marked left-sided gaze preference, hemidysesthesia, hemi-neglect, and aphasia. A head computed tomography (CT) scan excluded intracranial haemorrhage (Supp. Fig. S1). His baseline National Institute of Health Stroke Scale (NIHSS) score was 20, his Rapid Arterial Occlusion Evaluation (RACE) score was 8, and his Albert Stroke Programme Early CT score (ASPECTS) was 9.

Address for correspondence: Di Li, Department of Neurointervention, Dalian Municipal Central Hospital affiliated with Dalian Medical University, Dalian, China e-mail: jzlidi@126.com; Shen Li, Department of Neurology, Dalian Municipal Central Hospital affiliated with Dalian Medical University, Dalian, China, e-mail: listenlishen@hotmail.com

Received: 2.09.2020 Accepted: 8.03.2021 Early publication date: 14.04.2021 
Intravenous recombinant tissue plasminogen activator (rtPA) was administered followed by endovascular therapy with conscious sedation 150 minutes after the onset of symptoms.

A left common carotid artery (CCA) angiogram revealed a small stump at the origin of the left ICA, indicative of chronic occlusion (Fig. 1A). Perfusion into the MCA territory was not observed. Right CCA angiogram demonstrated insufficient leptomeningeal collaterals (ASTIN/SIR:2) reconstituted by a patent AComA (Fig. 1B). Left vertebral artery (VA) angiogram showed flow to the supraclinoid segment of the left ICA and MCA from the left PComA, and revealed an occlusion at the mid M1 segment of the left MCA (Fig. 1C). As the left PComA was $1.3 \mathrm{~mm}$ in diameter, a stent-retriever thrombectomy through the collateral PComA was selected. An $8 \mathrm{~F}$ guide catheter was placed in the left VA followed by a $5 \mathrm{~F}$ Navien catheter (intermediate catheter, 0.058 -inch diameter, Medtronic, Irvine, CA, USA) and a Rebar 18 microcatheter (0.021-inch diameter, Medtronic) that were coaxially advanced over a 0.014-inch microwire (Stryker, Kalamazoo, MI, USA) to the distal basilar artery (BA). Next, the microcatheter was advanced through the left P1 segment, then through the PComA into the M1 segment, and finally placed distal to the clot. A $4 \times 20 \mathrm{~mm}$ Solitaire FR stent (Medtronic) was then introduced through the microcatheter and fully deployed across the occluded left MCA (Fig. 1D). After the stent had been maintained in place for five minutes, the $5 \mathrm{~F}$ Navien catheter was advanced to the left posterior cerebral artery along the guidewire. The stent and microcatheter were slowly pulled back in the Navien catheter (Fig. 1E), and then withdrawn outside the body through the $8 \mathrm{~F}$ guide catheter. A large red thrombus was retrieved with a single pass (Fig. 1F). During clot retrieval, continuous manual aspiration with both the $5 \mathrm{~F}$ Navien catheter and the $8 \mathrm{~F}$ guide catheter was performed, using two 30-mL syringes. A subsequent angiogram revealed significant flow restoration [Thrombolysis in Cerebral Infarction (TICI) score $2 \mathrm{~b}$ in the left MCA (Fig. 1G)]. Time to revascularisation, defined as the time from the femoral access to the achievement of revascularisation, was 38 minutes.

The patient started to improve immediately after thrombectomy. Head CT following the procedure showed no haemorrhage. A follow-up head CT the next day revealed scattered acute infarction restricted to the MCA territory in the left basal ganglia, as well as the left frontal and temporal lobes, and did not show intracranial haemorrhage (Supp. Fig. S2). There were no new infarcts in the posterior circulation, which confirmed a lack of embolisation in the stent-retriever passing territory. Symptoms that persisted at that time included partial gaze preference, right face droop, right hemiparalysis, sensory aphasia, and dysarthria. The NIHSS score had fallen to 10 . A cervical and cerebral CT angiogram performed seven days later confirmed the recanalisation of the previously occluded left MCA (Fig. 1H) and a chronic occlusion at the origin of the right ICA with a small stump (Fig. 1I). At discharge, 10 days post stroke, the patient's symptoms were sensory aphasia and right facial droop. His NIHSS score was 3. At the four-week follow-up visit, patient recovery was nearly complete, with only the right facial droop persisting. His NIHSS score was 2 and modified Rankin Scale (mRS) score was 0 . Written informed consent was obtained from the patient.

Nearly $20 \%$ of ischaemic strokes are related to severe extracranial carotid lesions, $10 \%$ of which are caused by carotid occlusion [7]. Regardless of the mechanism of ICA occlusion, symptoms are usually caused by a coexisting intracranial occlusive embolus, commonly in the middle cerebral artery (MCA) or carotid terminus [7]. Therefore, in most patients the final outcome depends on timely restoration of distal intracranial flow, rather than recanalisation of the proximal occlusion $[8,9]$. Using a collateral vascular approach may provide an alternative quick, safe, and effective endovascular treatment for acute stroke patients presenting a large intracranial vessel occlusion without a direct route to the occlusion site. The first successful cross-circulation aspiration thrombectomy via the patent PComA was for an acute MCA occlusion [3]. Then, an anterior-posterior revascularisation of a BA occlusion upon intra-arterial thrombolysis and aspiration thrombectomy through a patent PComA was reported [4]. Kim et al. described a cross-circulation stent-retriever thrombectomy via the patent PComA for acute MCA ischaemic stroke with chronic cervical ICA occlusion [2]. More recently, two cases of trans-AComA stent-retriever thrombectomy were successfully carried out for acute MCA occlusion tandem with ICA occlusion [5]. These cases demonstrate the feasibility of cross-circulation thrombectomy.

To date, there has been no imaging approach that can accurately distinguish acute from chronic ICA occlusions, both of which can establish collateral circulation at the distal end of the occlusion. Recently, Hasan et al. analysed and classified the radiographic findings of 100 chronic ICA occlusion patients into four types with the aim of predicting the feasibility and safety of ICA revascularisation via endovascular therapy [10]. The occlusive morphology and relatively sound primary collateral circulation of our patient supported the diagnosis of chronic ICA occlusion. Moreover, our patient had type C occlusion in Hasan's classification, which has a low recanalisation rate and a high perioperative complication rate [10].

Recanalisation of a chronically occluded cervical carotid before treatment of the intracranial lesion might delay the time to distal recanalisation and potentially lead to a less favourable outcome [11]. In addition, this procedure carries significant risks, including distal embolisation into previously healthy intracranial vessels [e.g. anterior cerebral artery (ACA), carotid terminus], dissection or perforation of the extracranial carotid, and cerebral reperfusion haemorrhage (particularly after thrombolysis or when antiplatelet agents are administered after stent placement).

In our case, symptoms were mainly caused by thrombus or atherosclerotic plaque detachment from the occluded ICA stump and blockage of the left MCA, rather than cerebral 

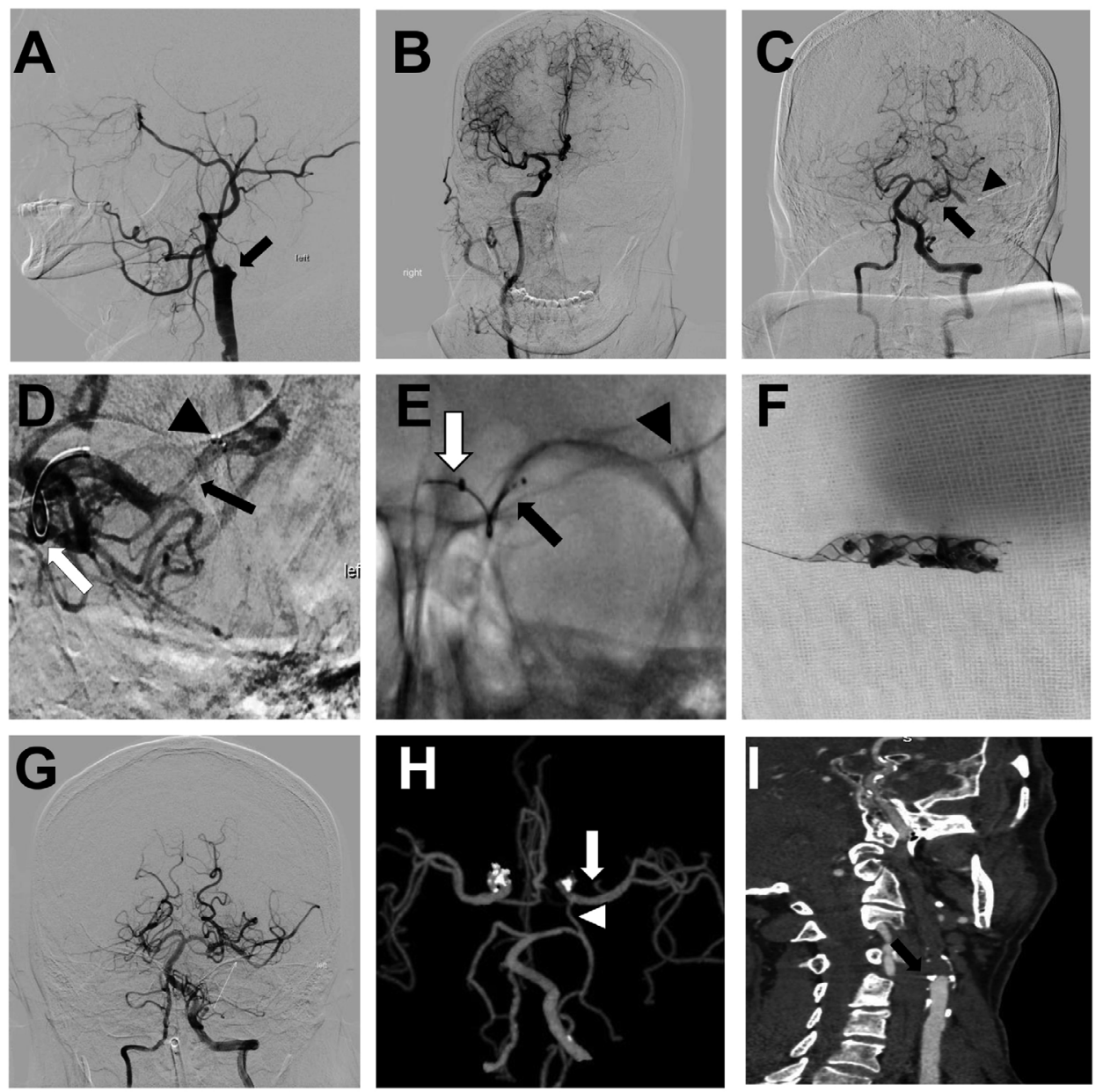

Figure 1. Brain images of patient suffering from acute left MCA occlusion. A. Lateral left CCA angiogram shows chronic occlusion at origin of left ICA, with small stump (arrow). B. Angiogram of right CCA (anterior-posterior) shows insufficient flow through leptomeningeal collaterals into left hemisphere reconstituted by patent AComA. C. Angiogram of left VA (anterior-posterior) reveals occlusion in mid M1 segment of left MCA (arrowhead) reconstituted by patent left PComA with diameter of $1.3 \mathrm{~mm}$ (arrow). D. A $4 \times 20 \mathrm{~mm}$ Solitaire FR stent retriever (black arrow) was deployed over whole length of thrombus in left MCA through left PComA (white arrow). Immediate antegrade flow to left MCA was restored. Note filling defect in M1 segment representing trapped thrombus within stent struts (black arrows). Stent has three radiopaque distal markers (black arrowhead). E. Stent retriever shown in mid portion of MCA (black arrowhead), microcatheter in proximal segment of MCA (black arrow), and 5F Navien catheter in left PCA (white arrow). F. Red clot retrieved using Solitaire FR stent. G. Angiogram of left VA (anterior-posterior) after thrombectomy shows significant restoration of flow (TICl score: $2 \mathrm{~b}$ ) in left MCA. H. Cervical and cerebral CT angiogram performed seven days after thrombectomy confirmed our previous observations: patent left PComA (white arrowhead), recanalisation of previously occluded left MCA (white arrow). I. Chronic occlusion at origin of right ICA with small stump (white arrow)

haemodynamic insufficiency caused by chronic ICA occlusion with poor collateral supply. Prompt reperfusion of viable tissue, and the prevention of subsequent expansion of cerebral infarction, are the keys to successful endovascular revascularisation in acute stroke [12]. Facing a chronic ICA occlusion, we believed that it was best to open the distal occlusion through the collateral pathway to save time. Nevertheless, we recognised that we had to try to open the ICA occlusion itself if the collateral pathway conditions did not allow a cross-circulation thrombectomy. We sought and evaluated alternative routes such as a wide patent AComA or PComA, and we found that the diameter of the PComA was $1.3 \mathrm{~mm}$, and thus suitable for the passage of the Rebar 18 microcatheter. Therefore, the opening of the left MCA through the patent PComA would save time, enhance the opening efficiency, and improve the clinical prognosis of the patient.

The cross-circulation technique is currently reserved for selected cases because of a high risk of complications, including dissection, perforation, escaping clots, and thromboembolic occlusions, associated with the advancement of endovascular devices through tortuous Circle of Willis collaterals with small diameters. This risk may be higher in patients with advanced atherosclerotic stenosis in the proximal vertebral and carotid arteries [6]. Newly developed microwires, microcatheters and clot-retrieval devices have been shown to improve the recanalisation rate and decrease complications [6]. 
The SWIM technique shortens the distance of the stent-retriever in the vascular cavity, which can further minimise the risk of proximal and distal embolisms, and mechanical damage to the vessel wall. In this case report, we placed a Navien, an intermediate catheter, through the BA to the ostium of the left posterior cerebral artery, and achieved recanalisation without complications. Large scale studies are necessary to verify the efficacy and safety of this approach.

Funding: This work was supported by the Natural Science Foundation of Liaoning Province (2019-MS-075), Liaoning Revitalisation Talents Programme (XLYC1807124), and Dalian Technology Innovation Programme (2019J13SN109). The sponsor had no role in the design or conduct of this research. Conflict of interest: The authors declare that the research was conducted in the absence of any commercial or financial relationships that could be considered as a potential conflict of interest.

Ethical approval: All procedures performed in studies involving human participants were in accordance with the ethical standards of the Ethical Committee of Dalian Municipal Central Hospital and with the 1964 Helsinki Declaration and its later amendments or comparable ethical standards.

Informed consent: Informed consent was obtained from all individual participants included in the study.

\section{References}

1. Rubiera M, Ribo M, Delgado-Mederos R, et al. Tandem internal carotid artery/middle cerebral artery occlusion: an independent predictor of poor outcome after systemic thrombolysis. Stroke. 2006; 37(9): 2301-2305, doi: 10.1161/01.STR.0000237070.80133.1d, indexed in Pubmed: 16888266.

2. Kim SK, Baek BH, Heo TW, et al. Successful Cross-circulation Stent-Retriever Embolectomy Through Posterior Communicating Artery for Acute MCA Occlusion by Using Trevo XP ProVue. Neurointervention. 2016; 11(1): 55-58, doi: 10.5469/neuroint.2016.11.1.55, indexed in Pubmed: 26958415.

3. Hui FK, Narayanan S, Cawley CM. Posterior-to-anterior circulation access using the Penumbra Stroke System for mechanical thrombectomy of a right middle cerebral artery thrombus. World Neurosurg. 2010; 73(1): 17-21, doi: 10.1016/j.surneu.2009.05.020, indexed in Pubmed: 20452865.

4. Liu W, Kung DK, Mahaney KB, et al. Anterior-to-posterior circulation approach for mechanical thrombectomy of an acutely occluded basilar artery using the penumbra aspiration system. World Neurosurg. 2012; 77(2): 398.E17-398.E20, doi: 10.1016/j. wneu.2011.04.025, indexed in Pubmed: 22120391.

5. Amuluru K, Romero CE, Pyle L, et al. Mechanical Thrombectomy of Acute Middle Cerebral Artery Occlusion Using Trans-Anterior Communicating Artery Approach. World Neurosurg. 2018; 112: 46-52, doi: 10.1016/j.wneu.2018.01.038, indexed in Pubmed: 29339323.

6-12. See supplementary references. 